\title{
Epidermal growth factor in the lungs of infants developing chronic lung disease
}

\author{
A.E. Currie, J.R. Vyas, J. MacDonald, D. Field, S. Kotecha
}

Epidermal growth factor in the lungs of infants developing chronic lung disease. A.E. Currie, J.R. Vyas, J. MacDonald, D. Field, S. Kotecha. C)ERS Journals Ltd 2001.

ABSTRACT: Growth factors important to lung growth and fibrosis have been poorly studied in chronic lung disease (CLD) of prematurity. Epidermal growth factor (EGF) promotes epithelial cell maturation, and vascular endothelial growth factor (VEGF) is important in angiogenesis.

The concentration of these growth factors was determined in 111 bronchoalveolar lavage fluid (BALF) samples from 35 ventilated infants: 13 developed CLD (median gestation 27 weeks, birthweight $820 \mathrm{~g}$ ), 16 developed and recovered from respiratory distress syndrome (RDS) (31 weeks, $1,415 \mathrm{~g}$ ) and six control infants (33 weeks, $2,075 \mathrm{~g}$ ) were ventilated for nonpulmonary reasons.

At birth, EGF in BALF from the CLD and RDS infants was lower than in the control infants (control versus $C L D, 7.3$ versus $0.0 \mathrm{pg} \cdot \mathrm{mL}^{-1}, \mathrm{p}<0.01$; control versus $\mathrm{RDS}, 7.3$ versus $5.0, \mathrm{p}=0.08)$. EGF increased in all groups with a more rapid increase in control infants. A close relationship was noted between BALF EGF and gestational age $(R=0.73)$. VEGF was undetectable at birth but increased at a similar rate in all three groups and did not correlate with gestation.

In conclusion, these data suggest that epidermal growth factor is closely correlated to gestation and that it may predispose preterm infants to develop chronic lung disease. Eur Respir J 2001; 18: 796-800.
Dept of Child Health, University of Leicester, Leicester, UK.

Correspondence: S. Kotecha, Dept of Child Health, University of Leicester, Leicester, LE2 7LX, UK.

Fax: 44 1162523282/2585502

Keywords: Bronchoalveolar lavage fluid

bronchopulmonary dysplasia

chronic lung disease of prematurity epidermal growth factor

growth factors

vascular endothelial growth factor

Received: October 92000

Accepted after revision July 52001

This study was funded in part by Action research and the British Lung Foundation.
Chronic lung disease (CLD) of prematurity remains a major cause of morbidity and mortality in preterm infants [1]. Recent advances including high frequency ventilation and nitric oxide have had a minimal impact on its incidence. Pulmonary inflammation appears to be an important risk factor for the development of CLD [2]. Inflammation is mediated by neutrophils and all agents associated with neutrophil recruitment and activation including proinflammatory cytokines, soluble adhesion molecules, chemokines, neutrophil products, e.g. elastase and reactive oxygen species, have all been reported to be increased in infants who develop CLD [2]. Sufficient evidence now exists to implicate lung inflammation as contributing to the development of CLD.

Inflammation is followed by resolution, repair and remodelling. Growth factors such as platelet-derived growth factor, insulin-like growth factors and transforming growth factor- $\beta$ promote synthesis and deposition of the extracellular matrix and others such as epidermal growth factor (EGF) and vascular endothelial growth factor (VEGF) promote proliferation and maturation of epithelial and endothelial cells respectively. EGF is a small polypeptide $(6 \mathrm{kDa})$ of 53 amino acid residues [3] and promotes mitogenesis and growth of all epithelial cell types. Increasing evidence supports its role in normal lung development and in maturation of epithelial cells in late gestation [4-6]. In rat and human lung explants, EGF has been shown to increase surfactant phospholipid synthesis [7] and production of surfactant protein A [8], respectively. Administration of EGF to foetal rabbits and nonhuman primates accelerates lung maturation with decreased respiratory disease in the newborn animal [9]. EGF clearly has a role to play in normal lung development and may also have a role to play in CLD where large areas of the epithelial barrier are injured.

Similarly, VEGF, a glycoprotein of $45 \mathrm{kDa}$, is a specific endothelial cell mitogen which has been shown to be increased in hypoxia [10]. It also increases vascular permeability. Although its messenger ribonucleic acid (mRNA) is also present in other cell types, especially epithelial cells and smooth muscle cells, its site of action is specific to endothelial cells. VEGF appears to play a role in the remodelling of the pulmonary arteries after birth and in resolution of acute lung injury [11]. Thus VEGF is an important factor for vascular maturation and may play a role in the repair processes that follow the lung inflammation that has been reported in CLD [2].

As the epithelial barrier is often injured in CLD, EGF would be expected to be increased in the reparative phase of CLD. Similarly, VEGF may be important in remodelling of the endothelium, especially in the pulmonary arteries, thus both growth factors would be expected to be increased in the lungs of infants who developed CLD. This study focused on EGF and VEGF in bronchoalveolar lavage fluid 
(BALF) obtained from infants who developed CLD, compared to infants who developed and recovered from respiratory distress syndrome (RDS) and to control infants requiring mechanical ventilation for nonpulmonary reasons.

\section{Methods}

\section{Patient groups}

Three groups of mechanically ventilated infants, who required mechanical ventilation at birth and who were admitted to the neonatal unit at the Leicester Royal Infirmary (Leicester, UK), were studied: 1) the CLD group, including infants with RDS who subsequently developed chronic lung disease, i.e. in whom a retrospective diagnosis was made on the basis of oxygen dependency at 28 days old together with an abnormal chest radiograph; 2) the RDS group contained infants with acute respiratory failure and chest radiograph changes consistent with RDS, who then recovered, that is, were nursed in air and had a normal chest radiograph by 28 days old; and 3 ) the control group, which included infants who received mechanical ventilation for nonpulmonary reasons.

Infants were excluded if there was documented or suspected evidence of infection at birth (i.e. with positive blood cultures) endotracheal tube secretion cultures or increased white cell count or C-reactive protein (CRP). Infants were also excluded if there were any congenital anomalies that may have influenced lung architecture (e.g. infants with congenital diaphragmatic hernia, tracheo-oesophageal fistula or cystic adenomatous malformations). None of the infants had received postnatal corticosteroids during the study period. Informed written consent was obtained from parents to perform bronchoalveolar lavage on infants whilst intubated and receiving mechanical ventilation. The study was approved by the local research ethics committee.

\section{Bronchoalveolar lavage}

Bronchoalveolar lavage (BAL) was performed using a nonbronchoscopic technique at the time of clinically indicated tracheal suctioning, as previously described [12-15]. Two aliquots of $1 \mathrm{~mL} \cdot \mathrm{kg}^{-1}$ (maximum $2 \mathrm{~mL}$ ) of saline were instilled and immediately aspirated. Infants were lavaged twice weekly for 4 weeks or until extubation, whichever occurred earlier. The collected BAL samples were placed on ice and centrifuged at $500 \times g$ at room temperature for $10 \mathrm{~min}$, within $10 \mathrm{~min}$ of collection. After centrifugation, the supernatant was collected and stored at $-70^{\circ} \mathrm{C}$ until VEGF and EGF were estimated.

Measurement of concentration of epidermal growth factor and vascular endothelial growth factor

The concentration of EGF and VEGF was measured with commercially available enzyme-linked immunosorbent assay (ELISA) kits according to the manufacturer's instructions (R\&D Systems, Abingdon, Oxfordshire, UK). The lowest sensitivity of the kits were $0.7 \mathrm{pg} \cdot \mathrm{mL}^{-1}$ and $9 \mathrm{pg} \cdot \mathrm{mL}^{-1}$, respectively, for EGF and VEGF. The calibration standard curves for all plates with each growth factor had an $\mathrm{R}^{2}$-value of $>0.97$.

\section{Statistics}

The concentration of both EGF and VEGF is presented as median values in $\mathrm{pg} \cdot \mathrm{mL}^{-1}$ for each diagnostic group against time. As recommended by the European Respiratory Society's guidelines for BAL in paediatrics including neonates [12], the data is presented as per $\mathrm{mL}$ of lavage fluid rather than per $\mathrm{mL}$ of epithelial lining fluid, since the estimation of the latter is inaccurate with current methods. Birth weight, gestation, fraction inspired oxygen, peak inspiratory pressure, oxygenation index and concentration of EGF and VEGF were compared between the three groups using the nonparametric analysis of variance (ANOVA), Kruskal-Walis and individual paired comparisons were made using the nonparametric Mann-Whitney U-test. A p-value $<0.05$ was considered significant.

\section{Results}

One-hundred and eleven BAL samples were obtained from 35 infants requiring mechanical ventilation. The patient characteristics are shown in table 1. From 29 infants developing respiratory failure, 13 infants developed chronic lung disease (CLD) (nine male and four female), and 16 developed and recovered from acute RDS (nine male and seven female). Six infants (five male, one female) requiring mechanical ventilation and inspired oxygen of $<28 \%$ were also studied (four for abdominal surgical reasons, one for mild asphyxia and one for apnoea). Antenatal corticosteroids were given to 12 (92\%) mothers of the CLD group, eight $(50 \%)$ of the RDS group and two (33\%) of the control group. Endotracheal natural surfactant was given to all but one infant in each of the CLD $(92 \%)$ and RDS (94\%) groups, whilst no infants in the control group received it.

Concentration of epidermal growth factor in bronchoalveolar lavage fluid

The concentration of EGF in BALF obtained from infants who subsequently developed CLD was undetectable immediately after birth in most infants, but increased gradually to $94.5 \mathrm{pg} \cdot \mathrm{mL}^{-1}$ by 21 days old (fig. 1). In contrast, in the RDS and control groups the EGF concentration was $5 \mathrm{pg} \cdot \mathrm{mL}^{-1}$ and $7.3 \mathrm{pg} \cdot \mathrm{mL}^{-1}$, respectively, immediately after birth. In both groups there was an increase in EGF with age, reaching $55.1 \mathrm{pg} \cdot \mathrm{mL}^{-1}$ by 21 days old in the RDS infants and $101.3 \mathrm{pg} \cdot \mathrm{mL}^{-1}$ by 10 days old in the 
Table 1. - Patient characteristics

\begin{tabular}{lccc}
\hline Characteristics & CLD & RDS & Control \\
\hline Subjects n & 13 & 16 & 6 \\
Sex M:F & $9: 4$ & $9: 7$ & $5: 1$ \\
Gestation weeks & $27(23-29)^{* * *}$ & $31(27-39)^{* * *}$ & $33(28-37)$ \\
Birthweight g & $820(630-1230)^{* * *}$ & $1415(840-4160)^{* * *}$ & $2075(1180-2520)$ \\
Antenatal corticosteroids & $12(92)$ & $8(50)$ & $2(33)$ \\
Surfactant & $12(92)$ & $15(94)$ & $0(0)$ \\
Fraction inspired oxygen & $0.45(0.26-0.90)^{*}$ & $0.43(0.21-1.00)^{*}$ & $0.24(0.21-0.28)$ \\
Peak inspiratory pressure & $20.8(15-36)^{*}$ & $18.6(13-22)^{*}$ & $16.3(15-20)$ \\
Oxygenation index & $8.4(2.6-30.5)^{*}$ & $12.6(0.9-94.3)^{*}$ & $2.7(1.3-4.6)$ \\
\hline
\end{tabular}

Data presented as $\mathrm{n}$ (range) or $\mathrm{n}(\%)$ unless stated otherwise. The fraction inspired oxygen, peak inspiratory pressures and oxygenation index are values obtained at $12 \mathrm{~h}$ of age. CLD: chronic lung disease; RDS: respiratory distress syndrome; M: male; F: female; *: $\mathrm{p}<0.05$ when CLD and control and RDS and control, but not RDS and CLD compared; ***: $p<0.001$ when CLD versus control and CLD and RDS, but not RDS and control compared.

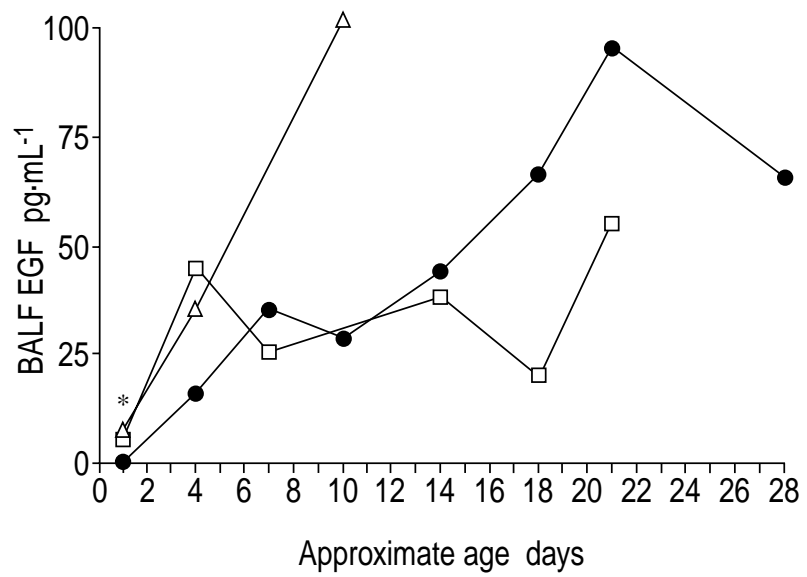

Fig. 1. - Epidermal growth factor (EGF) concentration in bronchoalveolar lavage fluid (BALF) is shown for infants requiring mechanical ventilation for respiratory failure and who recovered fully from respiratory distress syndrome (RDS: $\square$ ) or progressed to chronic lung disease (CLD) of prematurity (-), and infants who required mechanical ventilation for nonpulmonary reasons (control: $\triangle$ ). *: $\mathrm{p}<0.05$ when CLD group compared to control infants and to those infants who do not progress to CLD.

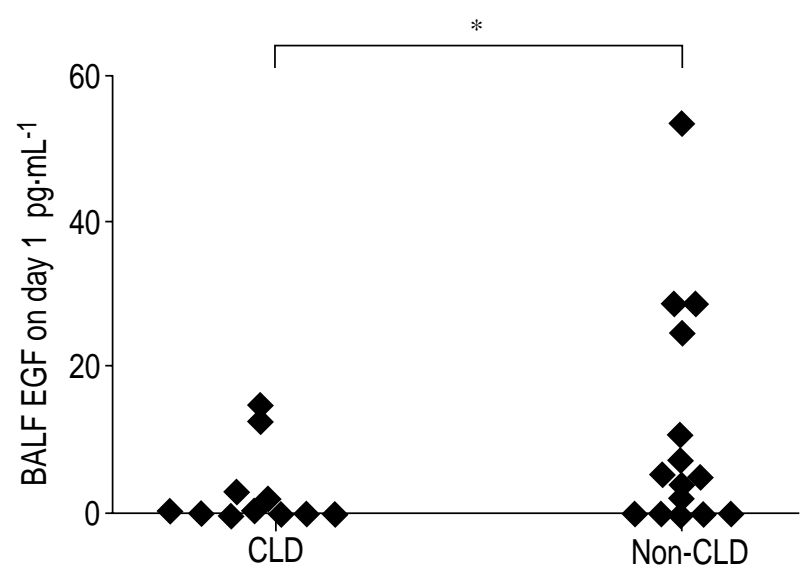

Fig. 2. - Concentration of epidermal growth factor (EGF) in bronchoalveolar lavage fluid (BALF) obtained from 1-day-old infants who developed chronic lung disease (CLD) of prematurity and those who did not progress to CLD. *: $\mathrm{p}<0.05$. control infants (fig. 1). Since decreased EGF may predispose the infant to respiratory failure, EGF concentration was compared in BALF from infants who did and did not develop CLD (fig. 2). Significant differences were found between the CLD infants and control infants $(\mathrm{p}<0.05)$, as well as between the CLD infants and those infants who did not develop CLD ( $<<0.05$, fig. 2). At 4 days old, the difference only approached significance when the CLD and non-CLD infants were compared $(\mathrm{p}=0.07)$.

As the most immature infants developed CLD, concentration of EGF was compared to both gestational age (fig. 3) and birth weight to determine whether a relationship existed. The concentration of EGF in the first lavage sample (which would reflect the infant's gestational age without postnatal influences) appeared to be related to gestational age $(r=0.73$, $\mathrm{p}<0.001)$ and a weaker relationship was noted for birth weight $(r=0.63, p<0.001)$.

Concentration of vascular endothelial growth factor in bronchoalveolar lavage fluid

In all three groups studied, VEGF concentration was undetectable in BALF obtained immediately after

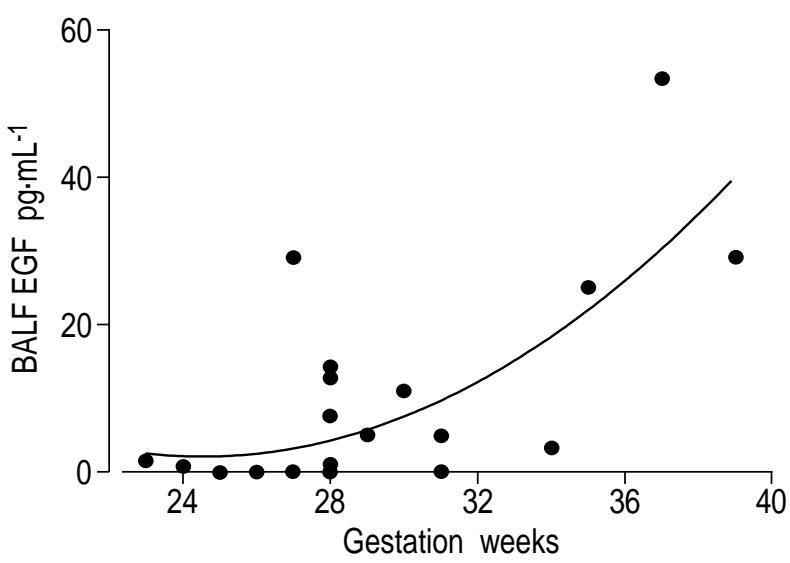

Fig. 3.-Epidermal growth factor (EGF) concentration in the first bronchoalveolar lavage fluid (BALF), obtained from ventilated infants recruited in the study, is related to the gestational age of the infant. A moderately good quadratic correlation was observed between EGF concentration and gestational age $(r=0.73, p<0.001)$. 


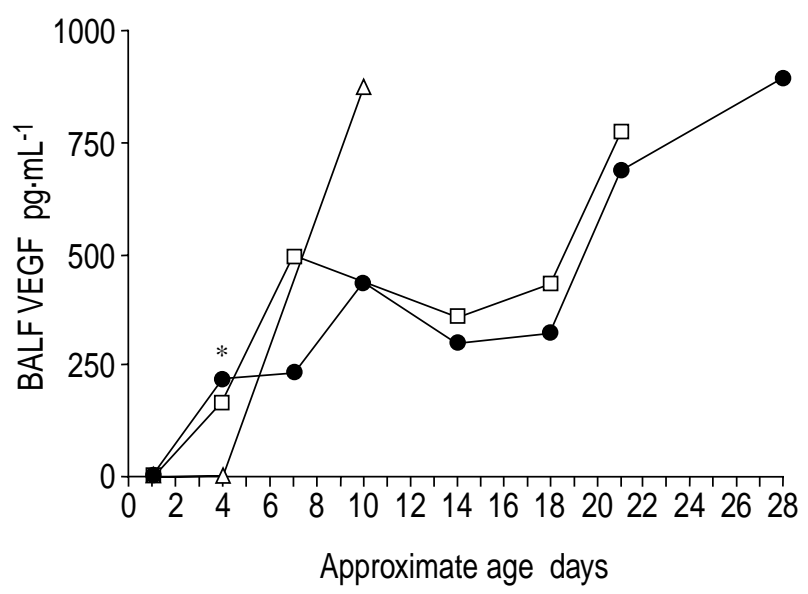

Fig. 4. - Vascular endothelial growth factor (VEGF) concentration in bronchoalveolar lavage fluid (BALF) is shown for infants requiring mechanical ventilation for respiratory failure and who recover fully from respiratory distress syndrome (RDS: $\square$ ) or progress to chronic lung disease (CLD) of prematurity $(\mathbf{O})$, and infants who required mechanical ventilation for nonrespiratory reasons (control: $\triangle$ ). *: $\mathrm{p}<0.05$ CLD versus controls and RDS versus controls.

birth, but increased thereafter (fig. 4). The VEGF concentration increased to $212 \mathrm{pg} \cdot \mathrm{mL}^{-1}$ in the CLD infants and $165.2 \mathrm{pg} \cdot \mathrm{mL}^{-1}$ in the RDS infants by 4 days old, but remained undetectable in the control group (CLD versus control $\mathrm{p}<0.05$, RDS versus control $\mathrm{p}<0.05)$. Thereafter, it increased gradually in the CLD and RDS groups to $679 \mathrm{pg} \cdot \mathrm{mL}^{-1}$ and $768 \mathrm{pg} \cdot \mathrm{mL}^{-1}$ respectively, but increased more rapidly in the control group to $873 \mathrm{pg} \cdot \mathrm{mL}^{-1}$ by 10 days old. There was no relationship between concentration of BALF, VEGF and gestational age or birthweight (data not shown).

\section{Discussion}

The present study investigated whether the growth factors for maturation of endothelial and epithelial cells are important in the pathogenesis of CLD. Both EGF and VEGF increased postnatally in all three groups, EGF was deficient at birth in infants who subsequently developed CLD when compared to, infants who developed and recovered from RDS, and to control infants. In addition, the concentration of EGF appeared to be closely related to both gestational age and birth weight. In contrast, VEGF increased in all three groups with time, but a maximum level was reached earlier in the control infants than in the RDS and CLD groups.

With improvements in neonatal care, the preterm infants are more likely to develop CLD than more mature infants who develop but recover from RDS. The relationship between gestation and EGF concentration is therefore interesting. EGF is known to promote maturation of type-II pneumocytes and subsequent production of components of surfactant $[7,8]$. It is interesting that CLD develops despite treatment with exogenous surfactant. In the present study, it was unclear whether the infants would have benefited from additional doses of surfactant given at a later stage e.g. at 5 days old or until the infants had synthesized sufficient endogenous surfactant. There is some evidence to suggest that additional doses of surfactant beyond the first few days of life may improve oxygenation [16].

In all three groups, EGF increased with time, probably reflecting a natural maturation of the infant exposed to the postnatal environment. The factors resulting in such an increase are likely to be the relatively hyperoxic postnatal environment as well as the natural increase of corticosteroids that occurs prior to delivery. Both antenatal and postnatal corticosteroids have been shown to promote maturation of type II cells [17]. ScotT et al. [18] reported increased urinary EGF in infants with CLD who responded to dexamethasone when compared to those who did not. In the present study, the infants had not received early postnatal corticosteroids; therefore, these were unlikely to be responsible for the increase in EGF. Antenatal corticosteroids are unlikely to have been responsible for the postnatal increase in the EGF, since all infants whose mothers had or had not received antenatal corticosteroids had similar increases postnatally.

In cell culture systems, EGF appears to accelerate the maturation of type II cells and the synthesis of components of the surfactant system [19]. In nonhuman primates, prenatal foetal treatment with EGF appears to decrease the incidence and severity of RDS by increasing maturation of type II pneumocytes and by the subsequent increase of surfactant [20]. In human infants it is recognized that most immature infants develop RDS and are at most risk of developing CLD. The present results show a relative deficiency of EGF in the first lavage sample obtained from infants, who subsequently developed CLD, and this corroborates evidence from animal models that implicate EGF as an important growth factor in the development of RDS. Whether strategies to increase EGF antenatally promote maturation of the foetal epithelium and better prepare the extremely preterm newborn infant to successful postnatal adaptation remains speculative.

Unlike EGF, no differences were found between the groups for BALF VEGF. However, like EGF, VEGF increased rapidly with time in agreement with previous reports of VEGF in preterm infants [21, 22]. VEGF has repeatedly been shown to increase with hypoxia. However, in addition to the relative hyperoxic postnatal environment, the newborn infant undergoes many other changes including a transformation from the foetal to "adult-type" circulation. It is conceivable that such pressure changes may exert a greater influence on VEGF production postnatally since mechanical shear stress forces have been shown to increase VEGF release [23].

In contrast to EGF, no differences could be detected in VEGF between the groups at birth, although a maximum concentration was achieved earlier in the control group (fig. 4). In addition, no differences between the concentration of VEGF in lung lavage fluid and either gestational age or birth weight were detected (data not shown). As VEGF has clearly been 
shown to increase vascular permeability it might be expected that this growth factor contributes to the protein deficiency that has been previously reported in infants who develop CLD [24].

In summary, the data suggests that epidermal growth factor was decreased at birth in infants who developed chronic lung disease. However, this decrease may simply reflect the immaturity of the infants who are at risk of developing chronic lung disease. A similar gestational effect was not seen for vascular endothelial growth factor. It was reassuring to note that infants at all gestations increased their pulmonary vascular endothelial growth factor and epidermal growth factor with time.

Acknowledgements. The authors would like to thank Action research for supporting A. Currie and the British Lung Foundation for supporting J. Vyas. The authors would also like to thank the staff and parents of babies on the neonatal unit for participating in this study.

\section{References}

1. Kotecha S, Silverman M. Chronic respiratory complications of neonatal disorders. In: Landau LI, Taussig LM, eds. Pediatric Respiratory Medicine. St. Louis, Mosby Inc., 1999; pp. 488-512.

2. Kotecha S. Cytokines in chronic lung disease of prematurity. Eur J Pediatr 1996; 155: Suppl. 2, S14 S17.

3. Soler C, Carpenter G. The epidermal growth factor family. In: Nicola NA, ed. Guidebook to Cytokines and Their Receptors. Oxford, Oxford University Press, 1994; pp. 194-197.

4. Ruocco S, Lallemand A, Tournier JM, Gaillard D. Expression and localization of epidermal growth factor, transforming growth factor-alpha, and localization of their common receptor in fetal human lung development. Pediatr Res 1996; 39: 448-455.

5. Stahlman MT, Orth DN, Gray ME. Immunocytochemical localization of epidermal growth factor in the developing human respiratory system and in acute and chronic lung disease in the neonate. $L a b$ Invest 1989; 60: 539-547.

6. Strandjord TP, Clark JG, Guralnick DE, Madtes DK. Immunolocalization of transforming growth factoralpha, epidermal growth factor (EGF), and EGFreceptor in normal and injured developing human lung. Pediatr Res 1995; 38: 851-856.

7. Gross I, Dynia DW, Rooney SA, et al. Influence of epidermal growth factor on fetal rat lung development in vitro. Pediatr Res 1986; 20: 473-477.

8. Whitsett JA, Pilot T, Clark JC, Weaver TE. Induction of surfactant protein in fetal lung. Effects of cAMP and dexamethasone on SAP-35 RNA and synthesis. J Biol Chem 1987; 262: 5256-5261.

9. Goetzman BW. Understanding bronchopulmonary dysplasia. Am J Dis Child 1986; 140: 332-334.

10. Ferrara N, Park JE. Vascular endothelial growth factor. In: Nicola NA, ed. Guidebook to Cytokines and Their Receptors. Oxford, Oxford University Press, 1994; pp. 232-234.

11. Watkins RH, D'Angio CT, Ryan RM, Patel A, Maniscalco WM. Differential expression of VEGF mRNA splice variants in newborn and adult hyperoxic lung injury. Am J Physiol 1999; 276: L858-L867.

12. de Blic J, Midulla F, Barbato Al, et al. Bronchoalveolar lavage in children. ERS Task Force on bronchoalveolar lavage in children. European Respiratory Society. Eur Respir J 2000; 15: 217-231.

13. Kotecha S, Wilson L, Wangoo A, Silverman M, Shaw RJ. Increase in interleukin (IL)-1 beta and IL-6 in bronchoalveolar lavage fluid obtained from infants with chronic lung disease of prematurity. Pediatr Res 1996; 40: 250-256.

14. Kotecha S, Chan B, Azam N, Silverman M, Shaw RJ. Increase in interleukin-8 and soluble intercellular adhesion molecule-1 in bronchoalveolar lavage fluid from premature infants who develop chronic lung disease. Arch Dis Child Fetal Neonatal Ed 1995; 72: F90-F96.

15. Kotecha S, Wangoo A, Silverman M, Shaw RJ. Increase in the concentration of transforming growth factor beta-1 in bronchoalveolar lavage fluid before development of chronic lung disease of prematurity. J Pediatr 1996; 128: 464-469.

16. Pandit PB, Dunn MS, Kelly EN, Perlman M. Surfactant replacement in neonates with early chronic lung disease. Pediatrics 1995; 95: 851-854.

17. Vyas J, Kotecha S. Effects of antenatal and postnatal corticosteroids on the preterm lung. Arch Dis Child Fetal Neonatal Ed 1997; 77: F147-F150.

18. Scott SM, Rogers C, Backstrom C. Dexamethasone therapy is associated with a rise in urinary epidermal growth factor concentrations in the preterm infant. Eur J Endocrinol 1995; 132: 326-330.

19. Solarin KO, Ballard PL, Guttentag SH, Lomax CA, Beers MF. Expression and glucocorticoid regulation of surfactant protein $\mathrm{C}$ in human fetal lung. Pediatr Res 1997; 42: 356-364.

20. Plopper CG, St. George JA, Read LC, et al. Acceleration of alveolar type II cell differentiation in fetal rhesus monkey lung by administration of EGF. Am J Physiol 1992; 262: L313-L321.

21. D'Angio CT, Maniscalco WM, Ryan RM, Avissar NE, Basavegowda K, Sinkin RA. Vascular endothelial growth factor in pulmonary lavage fluid from premature infants: effects of age and postnatal dexamethasone. Biol Neonate 1999; 76: 266-273.

22. Lassus P, Ristimaki A, Ylikorkala O, Viinikka L, Andersson S. Vascular endothelial growth factor in human preterm lung. Am J Respir Crit Care Med 1999; 159: 1429-1433.

23. Gan L, Miocic M, Doroudi R, Selin-Sjogren L, Jern S. Distinct regulation of vascular endothelial growth factor in intact human conduit vessels exposed to laminar fluid shear stress and pressure. Biochem Biophys Res Commun 2000; 272: 490-496.

24. Wang JY, Yeh TF, Lin YJ, Chen WY, Lin CH. Early postnatal dexamethasone therapy may lessen lung inflammation in premature infants with respiratory distress syndrome on mechanical ventilation. Pediatr Pulmonol 1997; 23: 193-197. 